\title{
On the Ulam-Hyers stability of a quadratic functional equation
}

\author{
Sang-Baek Lee ${ }^{1}$, Won-Gil Park ${ }^{2}$ and Jae-Hyeong Bae ${ }^{3^{*}}$
}

\footnotetext{
* Correspondence: jhbae@khu.ac.kr ${ }^{3}$ Graduate School of Education, Kyung Hee University, Yongin 446701, Republic of Korea Full list of author information is available at the end of the article
}

\section{Abstract}

The Ulam-Hyers stability problems of the following quadratic equation

$$
r^{2} f\left(\frac{x+y}{r}\right)+r^{2} f\left(\frac{x-y}{r}\right)=2 f(x)+2 f(y)
$$

where $r$ is a nonzero rational number, shall be treated. The case $r=2$ was introduced by J. M. Rassias in 1999. Furthermore, we prove the stability of the quadratic equation by using the fixed point method.

2010 Mathematics Subject Classification: 39B22; 39B52; 39B72.

Keywords: Hyers-Ulam stability, quadratic function

\section{Introduction}

In 1940, Ulam [1] proposed the general Ulam stability problem. In 1941, this problem was solved by Hyers [2] for the case of Banach spaces. Thereafter, this type of stability is called the Ulam-Hyers stability. In 1950, Aoki [3] provided a generalization of the Ulam-Hyers stability of mappings by considering variables. For more general function case, the reader is referred to Forti [4] and Găvruta [5].

Let $X$ be a real normed space and $Y$ be a real Banach space in the case of functional inequalities, as well as let $X$ and $Y$ be real linear spaces in the case of functional equations. The quadratic function $f(x)=c x^{2}(x \in \mathbb{R})$, where $c$ is a real constant, clearly satisfies the functional equation

$$
f(x+y)+f(x-y)=2 f(x)+2 f(y) .
$$

Hence, the above equation is called the quadratic functional equation. In particular, every solution $f: X \rightarrow Y$ of equation (1.1) is said to be a quadratic mapping. In 1983, Skof [6] obtained the first result on the Ulam-Hyers stability of equation (1.1).

In 1989, Aczel and Dhombres [7] obtained the general solution of Equation (1.1) for a function $f$ from a real linear space over a commutative field $F$ of characteristic 0 to the field F. In 1995, Kannappan [8] obtained the general solution of the functional equation

$$
f(\lambda x+y)+f(x-\lambda y)=\left(1+\lambda^{2}\right)[f(x)+f(y)] .
$$

The solution of the above equation is connected with bilinear functions. In 1995, Forti [9] obtained the result on the stability theorem for a class of functional equations

(C) 2011 Bae et al; licensee Springer. This is an Open Access article distributed under the terms of the Creative Commons Attribution License (http://creativecommons.org/licenses/by/2.0), which permits unrestricted use, distribution, and reproduction in any medium, provided the original work is properly cited. 
including Equation (1.1). It is also the first result on the Ulam-Hyers stability of the quadratic functional equation. Recently, Shakeri, Saadati and Park [10] investigated the Ulam-Hyers stability of Equation (1.1) in non-Archimedean $\mathcal{L}$-fuzzy normed spaces.

In 1996, Rassias [11] investigated the stability problem for the Euler-Lagrange functional equation

$$
f(a x+b y)+f(b x-a y)=\left(a^{2}+b^{2}\right)[f(x)+f(y)],
$$

where $a, b$ are fixed nonzero reals with $a^{2}+b^{2} \neq 1$. In 2009, Gordji and Khodaei [12] investigated the stability problem for the Euler-Lagrange functional equation

$$
f(a x+b y)+f(a x-b y)=2 a^{2} f(x)+2 b^{2} f(y),
$$

where $a, b$ are fixed integers with $a, b, a \pm b \neq 0$.

In this paper, we will investigate the Ulam-Hyers stability of the Euler-Lagrange functional equation as follows:

$$
r^{2} f\left(\frac{x+y}{r}\right)+r^{2} f\left(\frac{x-y}{r}\right)=2 f(x)+2 f(y)
$$

where $r$ is a nonzero rational number. Equation 1.4 is a special form with $a=b=\frac{1}{r}$ of Equation 1.2. Equation 1.4 is similar to Equation 1.3, but it is not a special form of Equation 1.3 since $a \neq b$ in Equation 1.3.

In 2009, Ravi et al. [13] obtained the general solution and the Ulam-Hyers stability of the Euler-Lagrange additive-quadratic-cubic-quartic functional equation

$$
\begin{aligned}
f(x+a y)+f(x-a y)=a^{2} f(x+y)+a^{2} f(x-y) & +2\left(1-a^{2}\right) f(x) \\
& +\frac{a^{4}-a^{2}}{12}[f(2 \gamma)+f(-2 \gamma)-4 f(y)-4 f(-\gamma)]
\end{aligned}
$$

for a fixed integer $a$ with $a \neq 0, \pm 1$. In [13], one can find the fact that Equation (1.1) implies Equation 1.5. Recently, $\mathrm{Xu}$, Rassias and $\mathrm{Xu}[14]$ investigated the stability problem for Equation 1.5 in non-Archimedean normed spaces. Euler-Lagrange type functional equations in various spaces have been constantly studied by many authors.

\section{Solution of the functional equation (1.4)}

Theorem 2.1 Let $r$ be a nonzero rational number and let $X$ and $Y$ be vector spaces. $A$ mapping $f: X \rightarrow Y$ satisfies the functional equation (1.4) if and only if it is quadratic.

Proof Suppose that $f$ satisfies Equation (1.4). Letting $x=y=0$ in (1.4), we gain $f(0)=$

0 . Putting $y=0$ in (1.4), we get

$$
r^{2} f\left(\frac{x}{r}\right)=f(x)
$$

for all $x \in X$. By (1.4) and the above equation, we have

$$
f(x+y)+f(x-y)=r^{2} f\left(\frac{x+y}{r}\right)+r^{2} f\left(\frac{x-y}{r}\right)=2 f(x)+2 f(y)
$$

for all $x, y \in X$. 
Conversely, suppose that $f$ is quadratic. Then we have

$$
f(r x)=r^{2} f(x)
$$

for all $x \in X$. Thus we obtain

$$
r^{2} f\left(\frac{x+y}{r}\right)+r^{2} f\left(\frac{x-y}{r}\right)=f(x+y)+f(x-y)=2 f(x)+2 f(y)
$$

for all $x, y \in X$. $\square$

Remark 2.2 Let $r$ be a nonzero real number and let $X$ and $\mathrm{Y}$ be vector spaces. Let $f$ : $X \rightarrow Y$ be a mapping satisfying the functional equation (1.4). By the same reasoning as the proof of Theorem 2.1, it is quadratic.

Remark 2.3 Let $r$ be a nonzero real number and let $\mathrm{X}$ and $\mathrm{Y}$ be vector spaces. Let $f$ : $X \rightarrow Y$ be a quadratic mapping and let, for all $x \in X$, the mapping $g_{x}: \mathbb{R} \rightarrow Y$ given by $g_{x}(t):=f(t x)(t \in \mathbb{R})$ be continuous. Then the mapping $f$ satisfies the functional equation (1.4).

\section{Stability of the quadratic equation (1.4)}

For $r=1$, the stability problem of Equation (1.4) has been investigated by Cholewa [15]. For $r=2$, the stability problem of Equation (1.4) has been proved by Rassias [16]. From now on, let $r$ be a nonzero rational number with $|r| \neq 2$.

In this section, we investigate the generalized Hyers-Ulam stability of the functional equation (1.4) in the spirit of Găvruta. Let $X$ be a normed space and $Y$ a Banach space. For a mapping $f: X \rightarrow Y$, we define a mapping $D f: X \times X \rightarrow Y$ by

$$
D f(x, y):=r^{2} f\left(\frac{x+y}{r}\right)+r^{2} f\left(\frac{x-y}{r}\right)-2 f(x)-2 f(y)
$$

for all $x, y \in X$. Assume that $\phi: X \times X \rightarrow[0, \infty)$ is a function satisfying

$$
\Phi(x, y):= \begin{cases}\sum_{k=1}^{\infty}\left(\frac{2}{r}\right)^{2 k} \varphi\left(\left(\frac{r}{2}\right)^{k} x,\left(\frac{r}{2}\right)^{k} y\right)<\infty \text { if } & |r|>2, \\ \sum_{k=0}^{\infty}\left(\frac{r}{2}\right)^{2 k} \varphi\left(\left(\frac{2}{r}\right)^{k} x,\left(\frac{2}{r}\right)^{k} y\right)<\infty \text { if } & |r|<2\end{cases}
$$

for all $x, y \in X$.

Lemma 3.1 Let a mapping $f: X \rightarrow Y$ satisfy $f(0)=0$ and the inequality

$$
\|D f(x, y)\| \leq \varphi(x, y)
$$

for all $x, y \in X$. Then

$$
\begin{cases}\left\|\left(\frac{2}{r}\right)^{2 n} f\left(\left(\frac{r}{2}\right)^{n} x\right)-f(x)\right\| \leq \frac{1}{4} \sum_{k=1}^{n}\left(\frac{2}{r}\right)^{2 k} \varphi\left(\left(\frac{r}{2}\right)^{k} x,\left(\frac{r}{2}\right)^{k} x\right) \text { if } & |r|>2 \\ \left(\frac{r}{2}\right)^{2 n} f\left(\left(\frac{2}{r}\right)^{n} x\right)-f(x) \| \leq \frac{1}{4} \sum_{k=0}^{n-1}\left(\frac{r}{2}\right)^{2 k} \varphi\left(\left(\frac{2}{r}\right)^{k} x,\left(\frac{2}{r}\right)^{k} x\right) \text { if } & |r|<2\end{cases}
$$

for all $n \in \mathbb{N}$ and $x \in X$.

Proof Let $|r|>2$. Now we are going to prove our assertion by induction on $n$. Replacing $y$ by $x$ in (3.3), we obtain

$$
\left\|\frac{r^{2}}{4} f\left(\frac{2}{r} x\right)-f(x)\right\| \leq \frac{1}{4} \varphi(x, x)
$$


for all $x \in X$. Replacing $x$ by $\frac{r}{2} x$ in (3.5) and multiplying $\frac{4}{r^{2}}$ to the resulting inequality, we have

$$
\left\|\frac{4}{r^{2}} f\left(\frac{r}{2} x\right)-f(x)\right\| \leq \frac{1}{r^{2}} \varphi\left(\frac{r}{2} x, \frac{r}{2} x\right)
$$

for all $x \in X$. Thus inequality (3.4) holds for $n=1$. We assume that the assertion is true for a fixed natural number $n$. Replacing $x$ by $\left(\frac{r}{2}\right)^{n} x$ in (3.6) and multiplying $\left(\frac{2}{r}\right)^{2 n}$ to the resulting inequality, we have

$$
\begin{aligned}
\left.\|\left(\frac{2}{r}\right)^{2(n+1)} f\left(\frac{r}{2}\right)^{n+1} x\right)-\left(\frac{2}{r}\right)^{2 n} & \left.f\left(\frac{r}{2}\right)^{n} x\right) \| \\
\leq & \left.\frac{1}{4}\left(\frac{2}{r}\right)^{2(n+1)} \varphi\left(\frac{r}{2}\right)^{n+1} x,\left(\frac{r}{2}\right)^{n+1} x\right)
\end{aligned}
$$

for all $x \in X$. Thus we have

$$
\begin{aligned}
\|\left(\frac{2}{r}\right)^{2(n+1)} & \left.f\left(\frac{r}{2}\right)^{n+1} x\right)-f(x) \| \\
\leq & \left.\left.\|\left(\frac{2}{r}\right)^{2(n+1)} f\left(\frac{r}{2}\right)^{n+1} x\right)-\left(\frac{2}{r}\right)^{2 n} f\left(\frac{r}{2}\right)^{n} x\right) \| \\
& \left.+\|\left(\frac{2}{r}\right)^{2 n} f\left(\frac{r}{2}\right)^{n} x\right)-f(x) \| \\
\leq & \left.\frac{1}{4} \sum_{k=1}^{n+1}\left(\frac{2}{r}\right)^{2 k} \varphi\left(\frac{r}{2}\right)^{k} x,\left(\frac{r}{2}\right)^{k} x\right)
\end{aligned}
$$

for all $x \in X$. Hence inequality (3.4) holds for all $n \in \mathbb{N}$.

The proof of the case $|r|<2$ is similar to the above proof. $\square$

In the following theorem we find that for some conditions there exists a true quadratic mapping near an approximately quadratic mapping.

Theorem 3.2 Assume that a mapping $f: X \rightarrow Y$ satisfies $f(0)=0$ and inequality (3.3). Then there exists a unique quadratic mapping $Q: X \rightarrow Y$ satisfying

$$
\|f(x)-Q(x)\| \leq \frac{1}{4} \Phi(x, x)
$$

for all $x \in X$.

Proof Let $|r|>2$. For each $n \in \mathbb{N}$, define a mapping $Q_{n}: X \rightarrow Y$ by $Q_{n}(x):=\left(\frac{2}{r}\right)^{2 n} f\left(\left(\frac{r}{2}\right)^{n} x\right)$ for all $x \in X$. For each $x \in X$, in order to prove the convergence of the sequence $\left\{Q_{n}(x)\right\}$,we have to show that $\left\{Q_{n}(x)\right\}$ is a Cauchy sequence in $Y$. By inequality (3.7), for all integers $l, m$ with $0 \leq l<m$, we get

$$
\begin{aligned}
\left.\left.\|\left(\frac{2}{r}\right)^{2 l} f\left(\frac{r}{2}\right)^{l} x\right)-\left(\frac{2}{r}\right)^{2 m} f\left(\frac{r}{2}\right)^{m} x\right) \| \\
\left.\leq \frac{1}{4} \sum_{n=l}^{m-1}\left(\frac{2}{r}\right)^{2(n+1)} \varphi\left(\frac{r}{2}\right)^{n+1} x,\left(\frac{r}{2}\right)^{n+1} x\right)
\end{aligned}
$$


for all $x \in X$. Taking $l, m \rightarrow \infty$ in the above in the above inequality, by inequality (3.2), we may conclude that the sequence $\left\{Q_{n}(x)\right\}$ is a Cauchy sequence in the Banach space $Y$ for each $x \in X$. This implies that the sequence $\left\{Q_{n}(x)\right\}$ converges for each $x \in$ $X$. Hence one can define a function $Q: X \rightarrow Y$ by

$$
\left.Q(x):=\lim _{n \rightarrow \infty}\left(\frac{2}{r}\right)^{2 n} f\left(\frac{r}{2}\right)^{n} x\right)
$$

for all $x \in X$. By letting $n \rightarrow \infty$ in (3.4), we arrive at the formula (3.8). Now we show that $Q$ satisfies the functional equation (1.4) for all $x, y \in X$. By the definition of $Q$,

$$
\begin{gathered}
\left\|r^{2} Q\left(\frac{x+y}{r}\right)+r^{2} Q\left(\frac{x-y}{r}\right)-2 Q(x)-2 Q(y)\right\| \\
\left.\left.=\lim _{n \rightarrow \infty}\left(\frac{2}{r}\right)^{2 n} \| r^{2} f\left(\frac{2}{r}\right)^{n} \frac{x+y}{r}\right)+r^{2} f\left(\frac{2}{r}\right)^{n} \frac{x-y}{r}\right) \\
\left.\left.\quad-2 f\left(\frac{r}{2}\right)^{n} x\right)-2 f\left(\frac{r}{2}\right)^{n} y\right) \| \\
\left.\leq \lim _{n \rightarrow \infty}\left(\frac{2}{r}\right)^{2 n} \varphi\left(\frac{r}{2}\right)^{n} x\left(\frac{r}{2}\right)^{n} y\right)=0
\end{gathered}
$$

for all $x, y \in X$. Hence $Q$ is quadratic by Theorem 2.1. It only remains to claim that $Q$ is unique. Let $Q^{\prime}: X \rightarrow Y$ be another quadratic mapping satisfying inequality (3.8). Since $Q$ and $Q^{\prime}$ are quadratic mapping, we can easily show that $Q\left(\left(\frac{r}{2}\right)^{n} x\right)=\left(\frac{r}{2}\right)^{2 n} Q(x)$ and $Q^{\prime}\left(\left(\frac{r}{2}\right)^{n} x\right)=\left(\frac{r}{2}\right)^{2 n} Q^{\prime}(x)$ for all $n \in \ell$ and all $x \in X$. Thus we see that

$$
\begin{aligned}
\| Q(x) & -Q^{\prime}(x) \| \\
\leq & \left.\left.\left.\left.\left(\frac{2}{r}\right)^{2 n} \| Q\left(\frac{r}{2}\right)^{n} x\right)-f\left(\frac{r}{2}\right)^{n} x\right)\left\|+\left(\frac{2}{r}\right)^{2 n}\right\| f\left(\frac{r}{2}\right)^{n} x\right)-Q^{\prime}\left(\frac{r}{2}\right)^{n} x\right) \| \\
\leq & \left.\frac{1}{2}\left(\frac{2}{r}\right)^{2 n} \Phi\left(\frac{r}{2}\right)^{n} x\left(\frac{r}{2}\right)^{n} x\right)
\end{aligned}
$$

for all $n \in \mathbb{N}$ and all $x \in X$. By letting $n \rightarrow \infty$, we get that $Q(x)=Q^{\prime}(x)$ for all $x \in X$. The proof of the case $|r|<2$ is similar to the above proof. $\square$

Corollary 3.3 Let $|r|>2$ and let $\varepsilon, p, q \in \mathbb{N}$ with $p, q<2$ and $\varepsilon \geq 0$. If a mappingf: $X \rightarrow Y$ satisfies $f(0)=0$ and the inequality

$$
\|D f(x, y)\| \leq \varepsilon\left(\|x\|^{p}+\|y\|^{q}\right)
$$

for all $x, y \in X$, then there exists a unique quadratic mapping $Q: X \rightarrow Y$ such that

$$
\left.\|f(x)-Q(x)\| \leq \varepsilon \frac{\|x\|^{p}}{2^{p} r^{2-p}-4}+\frac{\|x\|^{q}}{2^{q} r^{2-q}-4}\right)
$$

for all $x \in X$.

Corollary 3.4 Let $|r|>2$ and let $\varepsilon, s, t \in \mathbb{R}$ with $s+t<2$ and $\eta \geq 0$. If a mapping $f$ : $X \rightarrow Y$ satisfies $f(0)=0$ and the inequality

$$
\|D f(x, y)\| \leq \eta\|x\|^{s}\|y\|^{t}
$$


for all $x, y \in X$, then there exists a unique quadratic mapping $Q: X \rightarrow Y$ such that

$$
\|f(x)-Q(x)\| \leq \frac{\eta\|x\|^{s+t}}{2^{s+t} r^{2-s-t}-4}
$$

for all $x \in X$.

Let $|r|>2$ and let $\varepsilon$ be a nonnegative real number. If a mapping $f: X \rightarrow Y$ satisfies $f$ (0) $=0$ and the inequality

$$
\|D f(x, y)\| \leq \eta
$$

for all $x, y \in X$, then there exists a unique quadratic mapping $Q: X \rightarrow Y$ such that

$$
\|f(x)-Q(x)\| \leq \frac{\eta}{r^{2}-4}
$$

for all $x \in X$.

Corollary 3.5 Let $|r|<2$ and let $\varepsilon, p, q \in \mathbb{R}$ with $p, q>2$ and $\varepsilon \geq 0$. If a mapping $f$ : $X \rightarrow Y$ satisfies $f(0)=0$ and the inequality

$$
\|D f(x, y)\| \leq \varepsilon\left(\|x\|^{p}+\|y\|^{q}\right)
$$

for all $x, y \in X$, then there exists a unique quadratic mapping $Q: X \rightarrow Y$ such that

$$
\left.\|f(x)-Q(x)\| \leq \varepsilon \frac{\|x\|^{p}}{4-2^{p} r^{2-p}}+\frac{\|x\|^{q}}{4-2^{q} r^{2-q}}\right)
$$

for all $x \in X$.

Corollary 3.6 Let $|r|<2$ and let $\varepsilon, s, t \in \mathbb{R}$ with $s+t>2$ and $\eta \geq 0$. If a mapping $f$ : $X \rightarrow Y$ satisfies $f(0)=0$ and the inequality

$$
\|D f(x, y)\| \leq \eta\|x\|^{s}\|y\|^{t}
$$

for all $x, y \in X$, then there exists a unique quadratic mapping $Q: X \rightarrow Y$ such that

$$
\|f(x)-Q(x)\| \leq \frac{\eta\|x\|^{s+t}}{4-2^{s+t} r^{2-s-t}}
$$

for all $x \in X$.

Let $|r|<2$ and let $\eta$ be a nonnegative real number. If a mapping $f: X \rightarrow Y$ satisfies $f$ (0) $=0$ and the inequality

$$
\|D f(x, y)\| \leq \eta
$$

for all $x, y \in X$, then there exists a unique quadratic mapping $Q: X \rightarrow Y$ such that

$$
\|f(x)-Q(x)\| \leq \frac{\eta}{4-r^{2}}
$$

for all $x \in X$.

\section{Stability using alternative fixed point}

In this section, we will investigate the stability of the given quadratic functional equation (3.1) using alternative fixed point. Before proceeding the proof, we will state the theorem, alternative fixed point. 
Theorem 4.1 (The alternative fixed point $[17,18])$ Suppose that we are given a complete generalized metric space $(\Omega, d)$ and a strictly contractive mapping $T: \Omega \rightarrow \Omega$ with Lipschitz constant L. Then (for each given $x \in \Omega$ ), either $d\left(T^{n} x, T^{n+1} x\right)=\infty$ for all $n \geq 0$ or there exists a natural number $n_{0}$ such that

(1) $d\left(T^{n} x, T^{n+1} x\right)<\infty$ for all $n \geq n_{0}$;

(2) the sequence $\left(T^{n} x\right)$ is convergent to a fixed point $y^{*}$ of $T$;

(3) $y^{*}$ is the unique fixed point of $T$ in the set $\Delta=\left\{y \in \Omega \mid d\left(T^{n_{0}} x, y\right)<\infty\right\}$;

(4) $d\left(y, y^{*}\right) \leq \frac{1}{1-L} d(y, T y)$ for all $y \in \Delta$.

From now on, let $\phi: X \times X \rightarrow[0, \infty)$ be a function

$$
\lim _{n \rightarrow \infty} \frac{\varphi\left(\lambda_{i}^{n} x, \lambda_{i}^{n} y\right)}{\lambda_{i}^{2 n}}=0 \quad(i=0,1)
$$

for all $x, y \in X$, where $\lambda_{i}=\frac{r}{2}$ if $i=0$ and $\lambda_{i}=\frac{2}{r}$ if $i=1$.

Theorem 4.2 Suppose that a mapping $f: X \rightarrow Y$ satisfies the functional inequality

$$
\|D f(x, y)\| \leq \varphi(x, y)
$$

for all $x, y \in X$ and $f(0)=0$. If there exists $L=L(i)<1$ such that the function

$$
x \mapsto(x):=\varphi(x, x)
$$

has the property

$$
(x) \leq L \cdot \lambda_{i}^{2} \cdot\left(\frac{x}{\lambda_{i}}\right)
$$

for all $x \in X$, then there exists a unique quadratic mapping $Q: X \rightarrow Y$ such that the inequality

$$
\|f(x)-Q(x)\| \leq \frac{L^{1-i}}{4(1-L)}(x)
$$

holds for all $x \in X$.

Proof Consider the set $\Omega:=\{g \mid g: X \rightarrow Y, g(0)=0\}$ and introduce the generalized metric $d$ on $\Omega$ given by

$$
d(g, h)=d(g, h):=\inf \{k \in(0, \infty) \mid\|g(x)-h(x)\| \leq k(x) \text { for all } x \in X\}
$$

for all $g, h \in \Omega$. It is easy to show that $(\Omega, d)$ is complete. Now we define a mapping $T: \Omega \rightarrow \Omega$ by $\operatorname{Tg}(x)=\frac{1}{\lambda_{i}^{2}} g\left(\lambda_{i} x\right)$ for all $x \in X$. Note that for all $g, h \in \Omega$,

$$
\begin{aligned}
d(g, h)<k & \Rightarrow\|g(x)-h(x)\| \leq k(x) \quad \text { for all } x \in X \\
& \Rightarrow \quad\left\|\frac{1}{\lambda_{i}^{2}} g\left(\lambda_{i} x\right)-\frac{1}{\lambda_{i}^{2}} h\left(\lambda_{i} x\right)\right\| \leq \frac{1}{\lambda_{i}^{2}} k \quad\left(\lambda_{i} x\right) \quad \text { for all } x \in X \\
& \Rightarrow \quad\left\|\frac{1}{\lambda_{i}^{2}} g\left(\lambda_{i} x\right)-\frac{1}{\lambda_{i}^{2}} h\left(\lambda_{i} x\right)\right\| \leq L k \quad(x) \quad \text { for all } x \in X \\
& \Rightarrow d(T g, T h) \leq L k .
\end{aligned}
$$


Hence we have that $d(T g, T h) \leq L d(g, h)$ for all $g, h \in \Omega$, that is, $T$ is a strictly contractive mapping of $\Omega$ with Lipschitz constant $L$.

We have inequality (3.6) as in the proof of Lemma 3.1. By inequalities (3.6) and (4.3) with the case $i=0$, we get

$$
\left\|\left(\frac{2}{r}\right)^{2} f\left(\frac{r}{2} x\right)-f(x)\right\| \leq \frac{1}{r^{2}}\left(\frac{r}{2} x\right) \leq \frac{1}{4} L(x)
$$

for all $x$, that is,

$$
d(f, T f) \leq \frac{L}{4}=\frac{L^{1}}{4}<\infty
$$

Similarly, we get

$$
d(f, T f) \leq \frac{1}{4}=\frac{L^{0}}{4}<\infty
$$

for the case $i=1$. In both cases we can apply the fixed point alternative and since $\lim _{n \rightarrow \infty} d\left(T^{n} f, Q\right)=0$, there exists a fixed point $Q$ of $T$ in $\Omega$ such that

$$
Q(x)=\lim _{n \rightarrow \infty} \frac{f\left(\lambda_{i}^{n} x\right)}{\lambda_{i}^{2 n}}
$$

for all $x \in X$. Letting $x=\lambda_{i}^{n} x, y=\lambda_{i}^{n} y$ in Equation (4.1) and dividing by $\lambda_{i}^{2 n}$,

$$
\|D Q(x, y)\|=\lim _{n \rightarrow \infty} \frac{D f\left(\lambda_{i}^{n} x, \lambda_{i}^{n} y\right)}{\lambda_{i}^{2 n}} \leq \lim _{n \rightarrow \infty} \frac{\varphi\left(\lambda_{i}^{n} x, \lambda_{i}^{n} y\right)}{\lambda_{i}^{2 n}}=0
$$

for all $x, y \in X$. That is, $Q$ satisfies Equation (1.4). By Theorem 2.1, $Q$ is quadratic. Also, the fixed point alternative guarantees that such $Q$ is the unique mapping such that $\|f(x)-Q(x)\| \leq k(x)$ for all $x \in X$ and some $k>0$. Again using the fixed point alternative, we have $d(f, Q) \leq \frac{1}{1-L} d(f, T f)$. Hence we may conclude that

$$
d(f, Q) \leq \frac{L^{1-i}}{4(1-L)}
$$

which implies inequality (4.4).

Corollary 4.3 Let $p, q, s, t$ be real numbers such that $p, q, s+t<2$ or $p, q, s+t>2$ and let $\varepsilon, \eta$ be nonnegative real numbers. Suppose that a mapping $f: X \rightarrow Y$ satisfies the functional inequality

$$
\|D f(x, y)\| \leq \varepsilon\left(\|x\|^{p}+\|y\|^{q}\right)+\eta\|x\|^{s}\|y\|^{t}
$$

for all $x, y \in X$ and $f(0)=0$. Then there exists a unique quadratic mapping $Q: X \rightarrow$ $Y$ such that the inequality

$$
\|f(x)-Q(x)\| \leq \frac{L^{1-i} \varepsilon}{4(1-L)}\left[\varepsilon\left(\|x\|^{p}+\|x\|^{q}\right)+\eta\|x\|^{s+t}\right]
$$

holds for all $x \in X$, where $L:=\max \left\{\lambda_{i}^{p}, \lambda_{i}^{q}, \lambda_{i}^{s+t-2}\right\}(i=0,1), \lambda_{0}=\frac{r}{2}$ if $p, q, s+t<2$; $\lambda_{1}=\frac{2}{r}$ if $p, q, s+t>2$. 


\section{Author details}

'Department of Mathematics, Chungnam National University, Daejeon 305-764, Republic of Korea ${ }^{2}$ Department of Mathematics Education, College of Education, Mokwon University, Daejeon 302-729, Republic of Korea ${ }^{3}$ Graduate School of Education, Kyung Hee University, Yongin 446-701, Republic of Korea

\section{Authors' contributions}

All authors contributed equally to this work. All authors read and approved the final manuscript.

\section{Competing interests}

The authors declare that they have no competing interests.

Received: 16 February 2011 Accepted: 6 October 2011 Published: 6 October 2011

\section{References}

1. Ulam, SM: A Collection of Mathematical Problems. Interscience Publishers, New York. 63 (1968)

2. Hyers, DH: On the stability of the linear functional equation. Proc Natl Acad Sci USA. 27, $222-224$ (1941). doi:10.1073/ pnas.27.4.222

3. Aoki, T: On the stability of the linear transformation in Banach space. J Math Soc Jpn. 2, 64-66 (1950). doi:10.2969/jmsj/ 00210064

4. Forti, GL: An existence and stability theorem for a class of functional equations. Stochastica. 4, 23-30 (1980). doi:10.1080/17442508008833155

5. Găvruta, P: A generalization of the Hyers-Ulam-Rassias stability of approximately additive mappings. J Math Anal Appl. 184, 431-436 (1994). doi:10.1006/jmaa.1994.1211

6. Skof, F: Proprietà locali e approssimazione di operatori. Rend Sem Mat Fis Milano. 53, 113-129 (1983). doi:10.1007/ BF02924890

7. Aczel, J, Dhombres, J: Functional Equations in Several Variables. Cambridge University Press, Cambridge (1989)

8. Kannappan, Pl: Quadratic functional equation and inner product spaces. Results Math. 27, 368-372 (1995)

9. Borelli, C, Forti, GL: On a general Hyers-Ulam stability result. Int J Math Math Sci. 18, 229-236 (1995). doi:10.1155/ S0161171295000287

10. Shakeri, S, Saadati, R, Park, C: Stability of the quadratic functional equation in non-Archimedean ?4?-fuzzy normed spaces. Int J Nonlinear Anal Appl. 1, 72-83 (2010)

11. Rassias, JM: On the stability of the general Euler-Lagrange functional equation. Demonstratio Math. 29, $755-766$ (1996)

12. Gordji, ME, Khodaei, $\mathrm{H}$ : On the generalized Hyers-Ulam-Rassias stability of quadratic functional equations. Abst Appl Anal 2009 (2009). Article ID 923476

13. Ravi, K, Rassias, JM, Arunkumar, M, Kodandan, R: Stability of a generalized mixed type additive, quadratic, cubic and quartic functional equation. J Inequal Pure Appl Math 10 (2009). Article ID 114

14. $\mathrm{Xu}, \mathrm{TZ}$, Rassias, JM $, \mathrm{Xu}, \mathrm{WX}$ : A fixed point approach to the stability of a general mixed AQCQ-functional equation in non-Archimedean normed spaces. Discrete Dyn Nat Soc 2010 (2010). Article ID 812545

15. Cholewa, PW: Remarks on the stability of functional equations. Aequationes Math. 27, 76-86 (1984). doi:10.1007/ BF02192660

16. Rassias, JM: On the stability of the multi-dimensional Euler-Lagrange functional equation. J Indian Math Soc (NS). 66 , 1-9 (1999)

17. Margolis, B, Diaz, JB: A fixed point theorem of the alternative for contractions on a generalized complete metric space. Bull Am Math Soc. 126, 305-309 (1968)

18. Rus, IA: Principles and Applications of Fixed point Theory, Ed. Dacia, Cluj-Napoca (1979) (in Romanian)

doi:10.1186/1029-242X-2011-79

Cite this article as: Lee et al:: On the Ulam-Hyers stability of a quadratic functional equation. Journal of Inequalities and Applications $20112011: 79$.

\section{Submit your manuscript to a SpringerOpen ${ }^{\circ}$ journal and benefit from:}

- Convenient online submission

- Rigorous peer review

- Immediate publication on acceptance

- Open access: articles freely available online

- High visibility within the field

- Retaining the copyright to your article

Submit your next manuscript at $>$ springeropen.com 